\title{
Acarology
}

\section{Fractionating the Plant Extract in Mini Volumes to Purify the Potential Antivirals from Terminalia Chebula}

\author{
Innokentii S. Solovarov ${ }^{1}$; Maxim A. Khasnatinov, $\mathrm{PhD}^{1 *}$; Tatyana A. Shishlyannikova \\ Natalie A. Lyapunova ${ }^{1}$; Galina A. Danchinova, $\mathrm{PhD}, \mathrm{ScD}^{1}$ \\ ${ }^{I}$ Scientific Center for Family Health and Human Reproduction Problems \\ ${ }^{2}$ Limnological Institute, SB RAS \\ Irkutsk, the Russian Federation
}

\begin{abstract}
Background: The preliminary experiments indicated that a 30\% aqueous extract of seeds of the Terminalia chebula has a significant neutralizing activity against tick-borne encephalitis virus (TBEV). In this study, we developed the most gentle approach to purify the antiviral substance.

Methods and Results: The extract of T. chebula seeds was fractionated using gel filtration in Sephadex G-200 and G-50. The fractions were tested in vitro for the presence of a direct antiviral action against TBEV and profiled using liquid chromatographymass spectrometry (LC-MS). Antiviral activity was detected in eight fractions from Sephadex G-200 and in three fractions from Sephadex G-50 gel filtration. The chromatogram of the most active Sephadex G-50 fraction exhibited three base peaks with a retention time of 3,5 and 25.5 minutes. The MS of individual peaks revealed seven compounds with mass-to-charge ratios 114.03; $279.16 ; 290.91 ; 301.15 ; 579.3 ; 354.03$, and 414.09 that were present at relatively high concentrations.

Conclusion: In spite of a small sample, it was possible to detect antiviral activity in the fractionated plant extract using convenient virological methods, as well as to separate the components of the single virus-neutralizing fraction using the LC-MS approach. The purity of resulting preparation needs to be improved. (International Journal of Biomedicine. 2019;9(1):57-60.)
\end{abstract}

Key Words: flavivirus $\bullet$ novel antiviral activity $\bullet$ chromatography $\bullet$ mass spectrometry

\section{Introduction}

Tick-borne encephalitis virus (TBEV) is one of the dangerous zoonotic pathogens transmitted to humans through the bite of hard ticks. The virus belongs to the Flaviviridae family. The disease causes damage to the nerve cells and their membranes, which leads to paralysis or even death of the patient. ${ }^{(1,2)}$ Currently, the only specific drug with antiviral activity against TBEV is a human immunoglobulin. This drug has several major drawbacks, including potential risk of contamination with human pathogens (e.g., hepatitis $\mathrm{C}$ virus or HIV), the demanding conditions of storage and transportation ("cold chain"), and limited availability of competent donorsall of which contribute to the high price and limited amount of

*Corresponding author: Maxim A. Khasnatinov, PhD. Scientific Center for Family Health and Human Reproduction Problems. Irkutsk, the Russian Federation.E-mail: khasnatinov@, gmail.com product. The efficacy of this drug is also disputable. Thus, the development of new antivirals is a very important scientific task. ${ }^{(3)}$ Plants are believed to be very promising sources of new medical substances. Having a big diversity of plants, it can be assumed that they can serve as a source of medicines for the treatment of most currently known infections. One of the brightest examples of plant-derived drugs is aspirin, also known as acetylsalicylic acid, which was originally isolated from willow (Salicaceae). The effectiveness of aspirin has been repeatedly demonstrated, as a pain medication (analgesic), an antipyretic, and an anti-inflammatory, as well as an inhibitor of platelet aggregation. ${ }^{(4)}$ In recent years, many other drugs possessing the antiviral, ${ }^{(5,6)}$ anti-inflammatory, ${ }^{(7)}$ antioxidant, ${ }^{(8)}$ antimicrobial, ${ }^{(9)}$ antifungal, ${ }^{(10)}$ anti-cancer, ${ }^{(11)}$ and other activities ${ }^{(12)}$ have been derived from plants.

Previously, ${ }^{(13)}$ we described a pronounced neutralizing activity against TBEV of aqueous extract of $T$. chebula seeds. To identify and characterize this neutralizing compound of the extract, it is necessary to purify it. However, the absence 
of any data on the physical and chemical properties of the substance (i.e. its molecular weight, chemical formula, or at least its concentration in the initial preparation) significantly complicates this task. In modern biochemical studies, one of the first steps in identifying biologically active molecules in multicomponent mixtures is molecular weight separation using column gel filtration. ${ }^{(14)}$ In this case, various versions of the Sephadex polymer are successfully used as the solid phase, and distilled water as the mobile carrier.

In this study, we attempted to fractionate the extract of $T$. chebula under the gentlest conditions. Besides this, the starting amount of the sample was minimized to reduce the background as much as possible.

Thus, the purpose of this study was to develop a purification technique and the concentration of an unknown component of $T$. chebula extract, which has a neutralizing activity on TBEV, using minimal amounts of the original preparation.

\section{Materials and Methods}

\section{The plant materials and preparation of the extract}

Dried and crushed to a fine powder state seeds of T. chebula were kindly provided by Dr. N. Oyuntsetseg (Department of Traditional Medicine, Mongolian State Medical University, Ulaanbaatar, Mongolia). To prepare the extract, $30 \mathrm{~g} \mathrm{~T}$. chebula seed powder were dissolved in 200 $\mathrm{ml}$ of sterile, double-distilled water and boiled at low heat for 15-30 minutes until the total volume of the mixture reached approximately $100 \mathrm{ml}$. The volume of the mixture was measured and brought to $100 \mathrm{ml}$ sterile, bidistilled water. Then the mixture was sequentially filtered through a sterile cloth filter, a sterile paper filter with a pore diameter of $0.45 \mu \mathrm{m}$ and sterilized by filtration through a vacuum $0.22 \mu \mathrm{m}$ filter. The resulting solution was considered as a $30 \%$ aqueous extract of seeds of T. chebula.

\section{Tick-borne encephalitis virus and cell culture}

We used an isolate $92 \mathrm{M}$ of TBEV of the Siberian subtype. ${ }^{(15)}$ Passaging of TBEV and determination of the concentration of infectious virus was carried out in the pig embryo kidney cell line (SPEV) purchased from the "Collection of human and animal cell lines for research in the field of virology" (FSI Influenza Research Institute, St. Petersburg, the Russian Federation). Cell culture was maintained on an RPMI1640 medium, supplemented with antibiotics and 5\% fetal calf serum (ThermoScientific, UK). The concentration of the infectious virus in the stock suspension and in the experiments on the neutralization of TBEV were determined by titration of plaque-forming units (PFU) in the cell culture of SPEV and expressed as a decimal logarithm of PFU per milliliter of suspension (lg PFU/ml). ${ }^{(16)}$

\section{Neutralization assay}

The neutralization reaction was performed according to E. Gould. ${ }^{(16)}$ In particular, $100 \mu \mathrm{l}$ of the virus suspension containing $1 \times 10^{5} \mathrm{PFU}$ of TBEV was mixed with $100 \mu \mathrm{l}$ of the test or control sample. Human donor immunoglobulin against TBEV (FSUS «SIC Microgen», Tomsk) at a concentration of 1 $\mathrm{mg} / \mathrm{ml}$ was used as a neutralization control. Sterile, bidistilled water was used as a reference control. The mixture of TBEV and the sample was incubated for $30 \mathrm{~min}$ at $37^{\circ} \mathrm{C}$ and then the concentration of the infectious virus was determined. The neutralization index was determined as the difference in titers (in logarithmic terms) of the test and reference samples. Each experiment was performed in three independent replications.

Column Gel Filtration

Gel filtration was performed under gravity force in a column of $1.2 \times 10 \mathrm{~cm}$ with water as a mobile phase. The column was packed with a $5 \%$ aqueous suspension of Sephadex G-50 or G-200 (Sigma-Aldrich, Sweden). Before loading the sample, the column was additionally equilibrated with $10 \mathrm{ml}$ of sterile bidistilled water. Afterwards $0.05 \mathrm{ml}$ of $30 \% \mathrm{~T}$. chebula seed extract were loaded onto the gel, and after the extract entered the column, $10 \mathrm{ml}$ of sterile bidistilled water was applied on top of the gel. Fractionation was performed at a flow rate of $0.5 \mathrm{ml} / \mathrm{min}$. The void volume of the column was considered equal to $6 \mathrm{ml}$, and eight fractions were collected with a volume of $4 \mathrm{ml}$ each. After that, the final fraction of 6 $\mathrm{ml}$ was collected.

\section{Liquid chromatography and mass spectrometry}

LC was performed using a Zorbax 300 SB-C18 reverse phase column with dimensions of $21 \times 150 \mathrm{~mm}$ and $5 \mu \mathrm{m}$ particle size and Agilent 1200 Series HPLC System. The separation was carried out in an acetonitrile gradient from $10 \%$ to $100 \%$ during 30 minutes, with the addition of a $0.1 \%$ heptafluorobutyric acid and a flow rate of $0.2 \mathrm{ml} / \mathrm{min}$. The readings were taken in the ultraviolet spectrum at wavelengths of $200 \mathrm{~nm}, 230 \mathrm{~nm}, 270 \mathrm{~nm}, 300 \mathrm{~nm}$, and $360 \mathrm{~nm}$. Electrospray ionization was performed using the Agilent 6210 TOF MS instrument in a positive mode, the ratio of mass and charge was estimated in the range of 70-3200 $\mathrm{m} \backslash \mathrm{z}$.

Presentation of results

The results were presented as the mean values of three independent replications. The standard deviation was used to evaluate the variability of data. The significance of differences was assessed using Student's t-test. Statistical analysis was performed using Microsoft EXCEL 2003. The LC-MS data were processed by the Agilent MassHunter Workstation software package (version B.01.03;Agilent Technologies Inc., USA).

\section{Results and Discussion}

\section{Fractionation of the extract}

The output of the colored part of the extract in Sephadex G-200 started with Fraction 4 and ended with the release of Fraction 8. In Sephadex G-50, the mobility of the colored part of the extract was one and a half times faster. The total volume of the eluate was $32 \mathrm{ml}$, which corresponded to a dilution of the initial sample by 640 times. Thus, the concentration of the extracted substances in each fraction approximately corresponded to a $0.05 \% T$. chebula extract. We have previously described that a $2 \% T$. chebula extract completely neutralizes infectious TBEV at a concentration of $1 \times 10^{5} \mathrm{PFU},{ }^{(13)}$ and the antiviral activity of the same extract against the influenza $A$ virus is detectable up to a concentration of $0.01 \%$. ${ }^{(17)}$ Thus, the concentration of the active substance in any of the fractions, even if it is evenly distributed over 
the entire volume of the column, should be sufficient for detectable neutralization of TBEV.

\section{Neutralizing activity of fractions}

After separation using the Sephadex G-200, the ability to neutralize TBEV was firstly detected in Fraction 2, just ahead of the colored fraction, reached a maximal values in Fractions 3 and 4 , and then minor virus inhibition activity was detected in each fraction until the final one (Fig.1A). To increase the resolution ability of the column, the Sephadex G-50 was used as well. The release of the neutralizing agent in this sorbent started with Fraction 4, reached the maximal concentrations in Fractions 5 and 6, and some virus neutralizing activity was also detected up to the final fraction (Fig.1B).

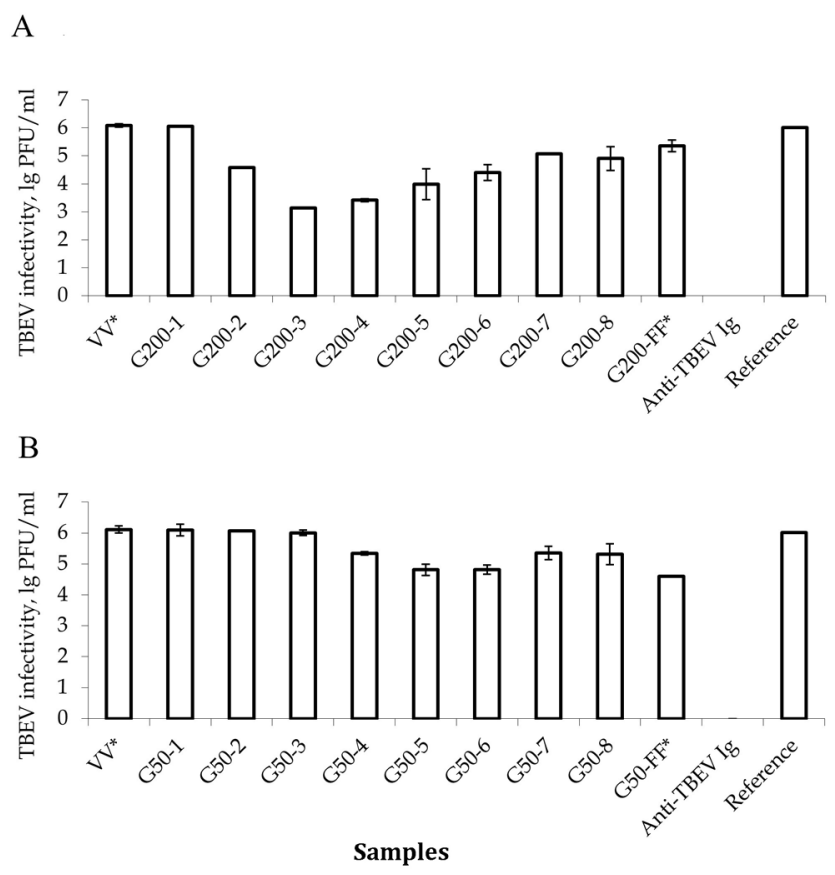

Fig. 1. Virus neutralizing activity of eight fractions (4 $\mathrm{ml}$ each) of T. chebula extract purification on A) Sephadex G-200 and B) Sephadex $G-50 ; * V V$ - void volume of the column, $F F$ - the final fraction (6 $\mathrm{ml}$ ); reference-sterile, double-distilled water.

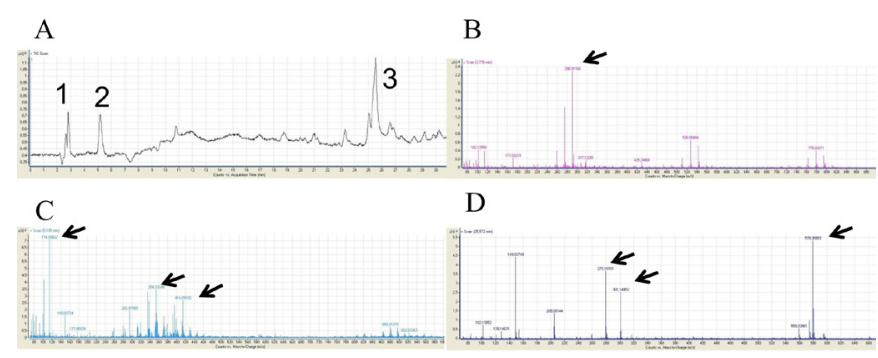

Fig. 2. Chromato-mass-spectrometry profile of Fraction 5 of T. chebula extract after separation on a Sephadex G-50 column. A) Chromatogram of total ion current (TIC) of Fraction 5; the time of collection is 30 minutes, the total concentration of the substance is indicated on the abscissa axis, the elution time of the peak from the column is indicated on the ordinate axis; 1, 2, 3-peaks of components of Fraction 5; B) The mass spectrum of peak 1; C) The mass spectrum of peak 2; D) The mass spectrum of peak 3. The arrows indicate the compounds with increased concentration.
The method of column gel filtration used in this study is based on the ability of substances with a higher molecular weight to pass between the Sephadex particles easily and migrate faster, whereas the substances with a lower molecular weight are able to penetrate into the particles of Sephadex and, as a result, migrate more slowly. The substances with a molecular weight exceeding the separation limit of the corresponding Sephadex polymer migrate in the void volume of the column. Therefore, upon completion of the gel filtration, the components of the extract with a high molecular weight accumulate in the earlier fractions, while the concentration of substances with low molecular weight is increased in the later fractions. According to the manufacturer's data, the exclusion limit is approximately $600 \mathrm{kDa}$ for Sephadex G-200 and $30 \mathrm{kDa}$ for Sephadex G-50. Since the entire diapason of the neutralizing activity was detected both in G-200 and in G-50, it can be assumed that the molecular weight of the target substance is less than $30 \mathrm{kDa}$.

\section{LC/MS spectrum of the fraction with a neutralizing effect}

Sephadex G-50 Fraction 5 possessing the highest neutralizing activity was used for LC followed by MS separation of the components of the extract of $T$. chebula. On the chromatographic profile, three peaks are visually identified with a retention time of 3,5 and 25.5 minutes (Fig. 2A). The mass spectra of each of these peaks indicated that neutralizing fraction includes about 30 individual components. Seven of these (values of $\mathrm{m} \backslash \mathrm{z} 114.03 ; 279.16 ; 290.91 ; 301.15 ; 579.3$; 354.03, and 414.09) are present in elevated concentrations (Fig. 2B-D). It is likely that a substance with a neutralizing activity against TBEV is either one of these seven components, or some of these components are derivatives (for example, fragments) of the analyzed substance.

In this study, a relatively small sample was used (initially only $30 \mathrm{~g}$ dry samples were extracted with $100 \mathrm{ml}$ of water, and only $50 \mu 1$ were used in the experiment). Nevertheless, it was possible to detect virus-neutralizing activity in separate fractions using convenient virological methods, as well as to separate the components of the single fraction, possessing antiviral activity, using the LC-MS approach. However, the purity of the resulting preparation seems to be insufficient for further analysis. Probably, additional purification methods are necessary besides the Sephadex-based gel filtration.

\section{Acknowledgments}

The authors are grateful to Dr. N. Oyuntsetseg(Department of Traditional Medicine, Mongolian State Medical University, Ulaanbaatar, Mongolia) for providing the powdered seeds of T. chebula, as well as to Ellina L. Manzarova and Valerii E. Salchak for technical assistance.

The LC-MS experiment was carried out at the Shared Research Facilities for Physical and Chemical Ultramicroanalysis LIN SB RAS.

\section{Competing Interests}

The authors declare that they have no competing interests. 


\section{References}

1. Burke DS, Monath TP. Flaviviruses. In: Knipe DM, Howley PM, editors. Fields Virology. Lippincott-Williams \& Wilkins, Philadelphia, PA; 2001:1043-1125.

2. Lindenbah BD, Rice CM. Molecular biology of flaviviruses. Adv Virus Res. 2003;59:23-61.

3. Prevention of tick-borne viral encephalitis. Sanitary rules SP3 1.3.2352-08. Moscow: Federal Service for Supervision of Consumer Rights Protection and Human Welfare; 2008. [In Russian].

4. Weiss HJ, Aledort LM. Impaired platelet/connectivetissue reaction in man after aspirin ingestion. Lancet. 1967;2(7514):495-7.

5. Lubbe A, Seibert I, Klimkait T, van der Kooy F. Ethnopharmacology in overdrive: the remarkable antiHIV activity of Artemisia annua. J Ethnopharmacol. 2012;141(3):854-9. doi:10.1016/j.jep.2012.03.024.

6. Cesar GZ, Alfonso MG, Marius MM, Elizabeth EM, Angel $\mathrm{CB}$, Maira HR, et al. Inhibition of HIV-1 reverse transcriptase, toxicological and chemical profile of Calophyllum brasiliense extracts from Chiapas, Mexico. Fitoterapia. 2011;82(7):102734. doi: 10.1016/j.fitote.2011.06.006.

7. Yu JS, Kim JH, Lee S, Jung K, Kim KH, Cho JY. Src/ Syk-Targeted Anti-Inflammatory Actions of Triterpenoidal Saponins from Gac (Momordica cochinchinensis) Seeds. Am J Chin Med. 2017:45(3):459-473. doi: 10.1142/ S0192415X17500288.

8. Intisar A, Zhang L, Luo H, Kiazolu JB, Zhang R, Zhang W. Anticancer constituents and cytotoxic activity of methanol-water extract of Polygonum bistorta L. Afr J Tradit Complement Altern Med. 2012;1:53-9. eCollection 2012.

9. Elisha IL, Botha FS, McGaw LJ and Eloff JN. The antibacterial activity of extracts of nine plant species with good activity against Escherichia coli against five other bacteria and cytotoxicity of extracts. BMC Complement Altern Med. 2017;17(1):133. Doi: 10.1186/s12906-017-1645-z.

10. Webster D, Taschereau P, Lee TD, Jurgens T. Immunostimulant properties of Heracleum maximum Bartr. J. Ethnopharmacol. 2006;106(3):360-3. doi:10.1016/j.jep.2006.01.018.

11. Yin SY, Yang NS, Lin TJ. Phytochemicals approach for developing cancer immunotherapeutics. Front Pharmacol. 2017;8:386. doi: 10.3389/fphar.2017.00386.

12. Myagmar BE, Aniya Y. Free radical scavenging action of medicinal herbs from Mongolia. Phytomedicine. 2000; 7(3):221-9.

13. Solovarov IS, Khasnatinov MA, Danchinova GA, Lyapunov AV, Bolotova NA, Manzarova EL, et al. [Assessment of neutralizing properties of DNA-aptamers and extracts of medicinal herbs against the tick-borne encephalitis virus]. Acta Biomedica Scientifica. 2017;2(1):84-88. [Article in Russian]. 14. Janiak M, Slavova-Kazakova A, Kancheva V, Amarowicz R. Sephadex LH-20 column chromatography of the hydrolysed lignan macromolecule of flaxseed. Bulgarian Chemical Communications. 2014;46(3):640-644.

15. Khasnatinov MA, Danchinova GA, Zlobin VI, Lyapunov AV, Arbatskaya EV, Chaporgina EA, et al. [Tick-borne encephalitis virus in Mongolia]. Siberian Medical Journal (Irkutsk). 2012;111(4):9-12. [Article in Russian].

16. Gould EA, Clegg JCS. Growth, titration and purification of togaviruses. In: Mahy BWJ, editor. Virology: A Practical Approach. IRL Press Ltd., Oxford; 1985:43-48.

17. Oyuntsetseg N, Khasnatinov MA, Molor-Erdene P, Oyunbileg Ja, Liapunov AV, Danchinova GA, et al. Evaluation of direct antiviral activity of the DEVA-5 herb formulation and extracts of five Asian plants against influenza A virus H3N8. BMC Complement Altern Med. 2014;14:235. doi: 10.1186/1472-6882-14-235. 